\title{
Supported discharge shortens hospital stay in patients hospitalized because of an exacerbation of COPD
}

\author{
E. Sala, L. Alegre, M. Carrera, M. Ibars, F.J. Orriols, M.L. Blanco, F. Cárceles, S. Bertran, F. Mata, \\ I. Font, A.G.N. Agustí
}

Supported discharge shortens hospital stay in patients hospitalized because of an exacerbation of COPD. E. Sala, L. Alegre, M. Carrera, M. Ibars, F.J. Orriols, M.L. Blanco, F.Cárceles, S. Bertran, F. Mata, I. Font, A.G.N. Agustí. (C) ERS Journals Ltd 2001.

ABSTRACT: This prospective, controlled, but not formally randomized study investigates the feasibility and efficiency of an alternative to standard hospitalization for patients with exacerbated chronic obstructive pulmonary disease (COPD), based upon supported discharge with nurse supervision at home.

Over a 12-month period, emergency physicians, not directly involved in the study, admitted 205 patients with exacerbated COPD to the authors' respiratory unit. Patients were included in the supported discharge group $(n=105)$ if they voluntarily chose to participate in the programme and lived in the city of Palma de Mallorca (where adequate home support could be provided). Patients not fulfilling these criteria (mainly residents outside the city) served as controls $(n=100)$. Inpatient treatment was standardized in all patients and included oxygen therapy, bronchodilators, antibiotics and steroids.

Both groups were comparable in terms of age (mean \pm SD: $70 \pm 10$ versus $65 \pm 11$ yr for supported discharge and control group, respectively), severity of airflow obstruction (forced expiratory volume in one second $45 \pm 18 \%$ reference versus $46 \pm 19 \%$ ref.), comorbidity and socioeconomic status. Length of hospital stay (LOS) in the supported discharge group was shorter $(5.9 \pm 2.8$ versus $8.0 \pm 5.1$ days, $p<0.001)$. After discharge, a respiratory nurse visited supported discharge patients at home during $7.3 \pm 3.8$ days. Only one patient $(1 \%)$ required hospital readmission during this period of time. The reduced LOS resulted in a lower utilization of hospital beds at any given point in time throughout the study period.

Within the framework and potential limitations of this study, the results indicate that the supported discharge programme in Spain: 1) allows a significant reduction in the length of hospital stay of patients hospitalized because of an exacerbation of chronic obstructive pulmonary disease; 2 ) does not result in an inappropriately increased rate of hospital readmissions; and 3) reduces the utilization of hospital resources.

Eur Respir J 2001; 17: 1138-1142.
Hospital Universitari Son Dureta, Palma de Mallorca, Spain.

\section{Correspondence: A. Agustí}

Servei Respiratori

Hospital Universitari Son Dureta

Andrea Doria 55

07014 Palma Mallorca

Spain

Fax: 34971175228

Keywords: Chronic bronchitis chronic obstructive pulmonary disease emphysema

health-care costs

managed care

Received: July 272000

Accepted after revision January 31 2001

This study was supported, in part, by FIS $(00 / 1011)$ and ABEMAR
Acute exacerbations of chronic obstructive pulmonary disease (COPD) are one of the leading causes of hospitalization in developed countries, particularly during the winter months when they often exceed the capacity of many hospitals $[1,2]$. Due to the important sanitary, social, legal and economic implications of this situation, there is an urgent need to find alternative strategies that can contribute to minimize it.

To this end, some studies have investigated if the provision of specialized, continuous and personalized health care at home, when the patient is clinically stable, can reduce or prevent these episodes of exacerbation [3, 4]. Others, on the contrary, have intended to avoid hospitalization when the patient is already exacerbated. These studies send the patient back home (with nursing support) immediately after formal assessment in a specialized respiratory unit in the hospital [5-7]. In the present study a third alternative was explored. It was hypothesized that patients with exacerbated COPD could be managed safely at home (with adequate nursing support) after the first few days of standard hospitalization, which is the period of time during which they really need an intravenous line, oxygen therapy and close medical supervision. If correct, this strategy should allow a significant reduction in their length of stay (LOS) (currently, 9-10 days in Spain), a more efficient use of hospital resources, and a potential reduction of the healthcare cost associated to their management. Secondarily, the results of this study may be also potentially useful to compare the efficiency of these alternatives to standard hospitalization in COPD in different countries. To date, all previous experiences in this field have been carried out in the UK [5-7]. Differences in healthcare systems (such as access to general practitioners and/or emergency room, for instance) may facilitate or jeopardize the implementation of these alternatives in different countries. 
This type of information is not yet available for comparison.

Accordingly, a prospective, controlled (although not formally randomized) study was designed to assess: 1) the feasibility and safety of the supported discharge programme; 2) its impact on the LOS of these patients; and 3) its effects on the use of hospital resources. This paper reports the results of the supported discharge programme after the first year of its implementation in the authors' institution, and compares them to those published recently in the UK.

\section{Methods}

\section{Patients and ethics}

Over a 12-month period (April 1999-April 2000), physicians not involved in the study who were working in the emergency room (ER) of the hospital, admitted 205 patients with a diagnosis of exacerbated COPD to the respiratory ward. Patients not responding to the initial therapy started in the ER either died or were admitted to the Intensive Care Unit (ICU) of the institution, none were included in the study. By definition, therefore, all the patients included in this study had responded favourably to the therapy started in the ER. Table 1 presents their main clinical and physiological characteristics. Once in the ward, patients were assigned to the supported discharge or the control group accordingly, mainly, to their site of residency (the city of Palma de Mallorca or somewhere else, respectively). This was because nursing support at home could be provided only for those patients living in Palma de Mallorca. Therefore, patients were included in the supported discharge group $(n=105)$ if they chose voluntarily to participate in the programme after being fully informed of its aims, nature and potential risks and lived in the city of Palma de Mallorca. The remaining patients $(n=100)$ did not fulfil these criteria (mainly because they lived outside the city, although three patients living in the city refused to receive home care) and served as controls. This project was approved by the Ethics Committee of the institution.

Table 1.-Clinical and physiological characteristics of the patients studied. There were no significant differences between the two groups

\begin{tabular}{lcc}
\hline & $\begin{array}{c}\text { Supported discharge } \\
\text { group }\end{array}$ & Control group \\
\hline Subjects n & 105 & 100 \\
$\mathrm{Age} \mathrm{yrs}$ & $70(44-87)$ & $66(39-88)$ \\
$\mathrm{Pa}, \mathrm{O}_{2} \mathrm{mmHg}$ & $57.1(32-100)$ & $56.7(30-88)$ \\
$\mathrm{Pa}, \mathrm{CO}_{2} \mathrm{mmHg}$ & $46.8(23-90.7)$ & $43.5(23-81.9)$ \\
$\mathrm{Arterial} \mathrm{pH}$ & $7.39(7.24-7.51)$ & $7.40(7.170-7.54)$ \\
$\mathrm{FEV} 1 \%$ ref & $45(20-76)$ & $46(15-73)$ \\
\hline
\end{tabular}

Arterial blood gases were obtained at the emergency room, breathing room air. Spirometry was obtained at discharge from hospital. $\mathrm{Pa}, \mathrm{O}_{2}$ : arterial oxygen tension; $\mathrm{Pa}_{\mathrm{a}} \mathrm{CO}_{2}$ : arterial carbon dioxide tension; $\mathrm{FEV}_{1}$ : forced expiratory volume in one second (reference values were those of a Mediterranean population [8]).

\section{Inpatient treatment}

All patients received standardized treatment that included: 1) aerosolized bronchodilators (ipratropium bromide $130 \mu \mathrm{g}$ and fenoterol $250 \mu \mathrm{g}$ four times daily); 2) systemic glucocorticoids (inpatient regimen: metilprednisolone $40 \mathrm{mg}$ three times daily for one day, $40 \mathrm{mg}$ twice daily one day and $40 \mathrm{mg}$ once daily up to hospital discharge. Home regimen: descending doses of oral prednisone for twelve days starting from $30 \mathrm{mg}$ ); 3) antibiotics (amoxycillin $875 \mathrm{mg}$ and clavulanic acid $175 \mathrm{mg}$ three times daily for seven days or clarythromycine $500 \mathrm{mg}$ twice daily for seven days); and, 4) controlled oxygen.

In all patients, both in the supported discharge programme and the control group, the decision to discharge the patient from hospital was taken according to the clinical judgement of the physicians in charge (experienced Spanish board certified pulmonary specialists). Such a judgement can not be quantified precisely; rather, it is a composite picture of the physiology of the patient (cardiac and breathing frequency, body temperature, arterial blood gases) as well as their willingness to accept discharge. All patients were discharged on this basis as soon as possible.

\section{The supported discharge programme}

Patients in the supported discharge programme were allowed to use nebulizers (Micron $\AA$, Medel Elettromedicali, Italy) and/or continuous $\mathrm{O}_{2}$ therapy at home if deemed necessary by the attending physicians. The day after hospital discharge, a specialized, hospital-based, clinical nurse visited them at home. Thereafter, home-visits were scheduled according to patient's needs. The programme nurse had daily meetings with the pulmonologists in the hospital to coordinate home-care needs. If necessary, during regular working hours the nurse could be reached by the patient (or the nurse could reach the doctor in the hospital) through a mobile phone. Likewise, if required, the nurse could send the patient back to hospital for immediate medical assessment by the pulmonary team (including new hospitalization if necessary).

At each visit at home, the nurse assessed the patient's general condition, shortness of breath intensity, and presence of cough or sputum production. Also, body temperature, heart rate, blood pressure, respiratory rate and arterial oxygen saturation $\left(\mathrm{Sa}_{\mathrm{a}} \mathrm{O}_{2}\right)$ (Nanox 2, Medlab $\mathrm{GmbH}$, Germany) were measured. At the end of the home support period, before being definitively discharged from the supported discharge programme, a lung specialist in the pulmonary clinic visited the patient.

\section{Data analysis}

To assess the viability and effectiveness of the supported discharge programme, several outcomes were defined a priori. These included: 1) LOS in 
hospital; 2) number of patients requiring hospitalization while still under the supervision of the supported discharge nurse at home (home-readmission's (HRA)); 3) number of patients requiring hospitalization during the first 2 weeks following final discharge from the supported discharge programme (early readmission's (ERA)); 4) number of patients requiring hospitalization after 2 weeks from final discharge of the supported discharge programme (late-readmission's (LRA)); and 5) mean number of hospital beds utilized daily by respiratory patients. Other variables included in the analysis were the number of nurse visits at home per patient and the number of mobile phone calls.

Results are expressed as mean \pm SD and range. The unpaired t-test and the Chi-squared test were used to compare quantitative and qualitative variables, respectively, in the supported discharge and control groups. A p-value $<0.05$ was considered statistically significant.

\section{Results}

Age, severity of respiratory failure determined on admission, and degree of airflow obstruction at discharge was not significantly different between the two study groups (table 1). All patients were current or exsmokers and all attended the emergency room of the authors' hospital because of acute worsening of their symptoms (mostly increased shortness of breath, cough and sputum production). All subjects included in the study (both in the supported discharge and control groups) had adequate familiar support at home and did not have significant comorbidity. At discharge, domiciliary oxygen therapy was prescribed in 41 patients $(39 \%)$ in the supported discharge programme and 14 patients $(14 \%)$ in the control group $(\mathrm{p}<0.005)$. Likewise, home nebulizers were prescribed in five patients $(4.8 \%)$ in the supported discharge programme and in one patient $(1 \%)$ in the control group.

LOS in the study group (supported discharge programme) was significantly shorter $(5.9 \pm 2.8$ days; range: $1-19$ days) than that of the control group $(8.0 \pm 5.1$ days; $1-30 ; \mathrm{p}<0.001)$. Unexpectedly, the latter was lower than that of historical controls hospitalized in the department also because of an exacerbation of COPD during 1998 (10.3 days). Patients included in the supported discharge programme were followed at home during $7.3 \pm 3.8$ days ( $1-17$ days). During this period of time, the mean number of nurse visits at home was $4.8 \pm 2.5(1-12)$ and the mean number of telephone calls among patients and nurses $2.3 \pm 2.0(0-8)$.

The majority of patients included in the study $(78.4 \%)$ were hospitalized only once during the 12 months of the study. Yet, 30 patients required hospitalization more than one time during this same period of time. As a result, the total number of hospital admissions (250) was higher than the total number of patients included in the study (205). Of these 250 admissions, 134 corresponded to the supported discharge programme and 116 to the control group. According to the classification discussed above (HRA,
ERA and LRA), these were distributed as follows in the study group: $1(0.7 \%)$ HRA, $3(2.2 \%)$ ERA and 25 $(18.7 \%)$ LRA. In the control group, by design, there were no HRA. Yet, $2(1.7 \%)$ were classified as (ERA) and $14(12.0 \%)$ as LRA. These values were not significantly different from those determined in the supported discharge group despite the fact that 46 patients included in the control group $(46 \%)$ lived outside of the city of Palma de Mallorca (or even outside of the island $(13 \%)$ ) and may have, therefore, been lost for follow-up should hospitalization have been needed.

Figure 1 shows a composite summary of three variables relevant to assess the impact of the supported discharge programme upon the use of hospital resources. Figure 1a presents the mean number of all patients hospitalized in the department (regardless of their diagnosis) every month during the study period (1999-2000) and the year before (1998-1999). Figure 1b shows the monthly average LOS of all these admissions during this same period of time. Finally, the figure 1c depicts the mean number of hospital beds used every month by patients with respiratory diseases under the care of the department, again during this same period of time. The arrow represents the point in time where the supported discharge programme was implemented. Figure 1 shows, firstly that the demand for hospitalization in the department was very similar before (total 2256 patients) and after the supported discharge programme had started (total 2294 patients); also, as expected, it is evident that there was a strong seasonal variability, the demand being significantly higher during the winter months. Secondly, despite this similar demand, the average LOS was lower during the study period than in the previous year (6.4 days versus 7.7 days on average, respectively). This change was particularly evident after the launch of supported discharge (April 1999). Finally, the net result of a similar hospitalization demand and a shorter LOS was a reduction in the average number of hospital beds used per day by respiratory patients.

\section{Discussion}

The presented results show that, in Spain, supported discharge: 1) is feasible, even for COPD patients with severe respiratory failure on admission, provided that they respond favourably to initial therapy; 2) allows a reduction of patients LOS and results in a lower number of hospital beds used at any given point in time (fig. 1); and; 3) does not cause a higher rate of ERA, as compared to the control group receiving standard care.

\section{Limitations of the study}

Two aspects of the study deserve comment. Firstly, it was not formally randomized. Instead patients were assigned to the supported discharge or control group based on their site of residency. Although this can theoretically bias the results, it is believed that this was 

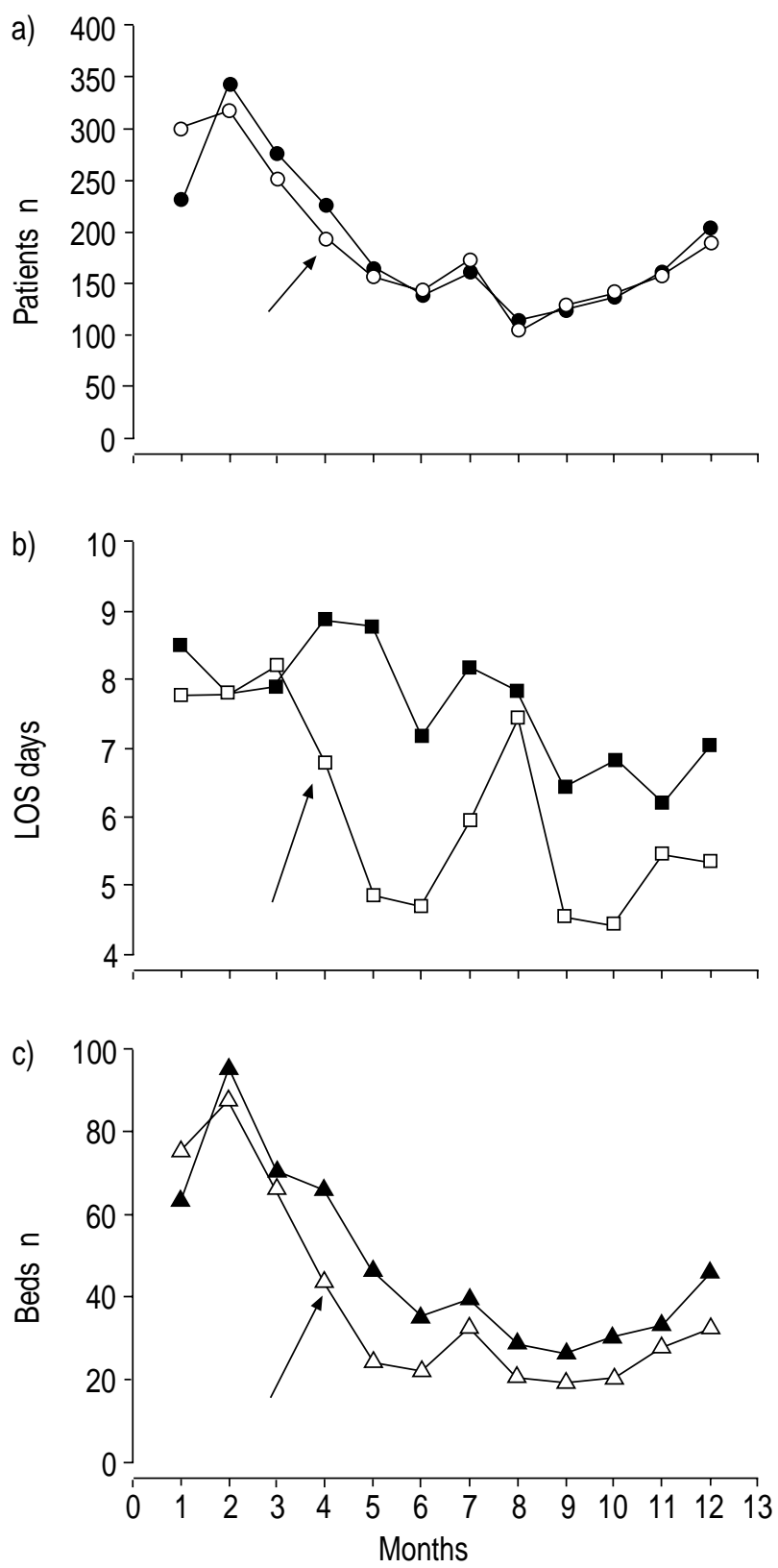

Fig. 1. - a) Number of patients admitted to the respiratory dept during $1998(\mathbf{O})$ and $1999(\bigcirc)$; b) length of hospital stay (LOS) during 1998 ( $\mathbf{\square})$ and 1999 ( $\square$ ). c) number of beds used by the respiratory department in $1998(\boldsymbol{\Delta})$ and in $1999(\triangle)$ data given as mean daily value. The arrow indicates the starting point of the supported discharge programme. For further explanations, see text.

not the case because, post hoc, both groups were similar in size, social circumstances and clinical and functional condition (table 1). Second, patients were discharged from hospital (in both groups) according to the "clinical judgement" of the attending physician (an experienced board certified pulmonary specialist in all cases). This approach, which is identical to that followed by some recent publications in the field $[6,7]$, is due to the fact that there are no internationally accepted, objective criteria that can guide clinicians in the discharge process of these patients.
Therefore, patients are normally discharged at a point in their recovery process where the physician thinks that the patient can finish it at home safely. Given that the supported discharge group was offered nurse supervision at home, it is likely that this point may have been reached earlier in this group than in the control group. However, this possibility is interpreted as, one of the main outcomes, rather than a limitation.

\section{Previous studies}

Over recent years, there has been a great deal of interest in exploring alternatives for the clinical management of patients with exacerbated COPD, mostly in UK centres [9]. These previous studies have shown that many patients can be successfully managed at home after formal assessment in a specialized respiratory unit in the hospital [5-7]. The presented study extends these previous observations by, first, exploring a different (intermediate) alternative for the management of these patients (supported discharge) and, second, by providing evidence about the feasibility of these type of alternative programmes outside the UK, where the different healthcare system peculiarities may facilitate (or limit) their implementation.

\section{Interpretation of findings}

With the limitations discussed above in mind, the study shows that the supported discharge programme allows a significant reduction in the LOS of patients hospitalized because of an exacerbation of COPD $(5.9 \pm 2.8$ versus $8.0 \pm 5.1$ days, supported discharge versus control group, respectively, $\mathrm{p}<0.001)$. This was not accompanied by a higher readmission rate but resulted in a lower number of hospital beds used at any given point in time (fig. 1). Several aspects of these results deserve comment.

First, the average LOS in the supported discharge programme was not very different from that reported recently (6.1 days) in the control group of a British study [7]. This may suggest that nursing support at home may not have been necessary for these patients. Whether or not this interpretation is correct is limited by the lack of objective criteria for hospital discharge discussed above. Further, it is likely that differences between countries in the way that these patients are managed in hospital may also contribute to explain this observation. Finally, despite the lack of formal randomization in the study, both groups received identical medical treatment and, nonetheless, LOS in the control group was significantly higher than in the supported discharge group. Therefore, it is believed that this is a true reflection of the effectiveness of the supported discharge programme. It was also of some interest to observe that, unexpectedly, the LOS of the control group was lower than that of historical controls hospitalized in the department because of an exacerbation of COPD during 1998 (10.3 days). It 
is thought that this is a reflection of a dynamic process of global improvement in the clinical management in COPD in the institution and, probably, in other institutions also (in the UK studies reported a similar trend [7]). Future studies will determine what is the minimum LOS of a patient hospitalized because of an exacerbation of COPD. The availability of a set of consensus criteria to discharge safely these patients (with or without nursing support at home) would be a valuable tool for such future studies.

Second, the efficacy of the supported discharge programme was assessed by analysing the rate of home HRA, ERA (2 weeks) and LRA ( $>2$ weeks). LRA are part of the natural history of the disease [10] and, therefore, not accountable to the efficiency of the supported discharge programme. In fact, LRA were not substantially different in between the supported discharge and control groups (18.7 versus $12 \%)$, particularly when the fact that $46 \%$ of the patients included in the control group lived outside of the city (and were, therefore, lost for follow-up) is taken into account. HRA, on the other hand, are due to patients showing poor clinical evolution at home. Whether this represents a failure of the supported discharge programme (as represented by the inclusion in the programme of patients too sick to be cared of at home) or, actually, an advantage of the programme (early detection of poor clinical evolution) is debatable. In any case, the percentage of HRA in the present study was very small (1\%). Finally, ERA probably indicate a true failure of the supported discharge programme. The percentage of ERA's in the supported discharge programme $(2.2 \%)$ was not statistically different from that seen in the control group $(1.7 \%)$ and lower than that reported by the study of GrAVIL et al. [5].

Finally, the economical savings of the supported discharge programme were not precisely quantified. However, it seems clear that it is not only feasible but also cost-effective because it reduced the mean number of hospital beds used by the respiratory department through the year (fig. 1c).

\section{Clinical implications}

The supported discharge programme was designed for patients hospitalized because of an exacerbation of COPD. By definition, therefore, all patients included in the present report were hospitalized with this diagnosis after showing a favourable response to the therapy started in the emergency room. The results of this study are applicable therefore to this type of patient only. However, the implementation of the programme demonstrated that it could also be used to support home care (and early discharge) in other types of respiratory diseases, such as bronchial asthma or community acquired pneumonia, with equally good results (data not shown). This experience, beyond the scope of the present study, should be taken into account when designing and implementing this type of clinical care strategies.

\section{Conclusions}

Patients hospitalized because of an exacerbation of chronic obstructive pulmonary disease can be safely discharged from hospital early during the course of their disease if adequate home supervision is provided. This shortens their hospital length of stay and contributes to optimizing the use of hospital resources.

Acknowledgements. The authors are grateful to A. Ita, L. Laosa and M. Rojals for their excellent work caring for the patients at home, and to all of the nursing staff of the Respiratory Unit for their loving care of the hospitalized patients, and to the physicians of the Emergency Room of the Hospital Universitari Son Dureta for their help and cooperation during the study.

\section{References}

1. Siafakas NM, Vermeire P, Pride NB, et al. Optimal assessment and management of chronic obstructive pulmonary disease (COPD). European Respiratory Society consensus statement. Eur Respir $J$ 1995; 8: $1398-1420$.

2. Montemayor T, Alfajame I, Escudero C, Morera J, Sánchez L. Normativa sobre diagnostico y tratamiento de la enfermedad pulmonar obstructiva crónica. Arch Bronconeumol 1996; 32: 285-301.

3. Güell R, Gonzalez A, Morante F, et al. Mejor en casa: un programa de asistencia continuada para los pacientes con enfermedad respiratoria crónica avanzada. Arch Bronconeumol 1998; 34: 541 - 546.

4. Campbell M, Stockdale-Woolley R, Nair S. An innovative home care program for the patient with chronic obstructive pulmonary disease. Chest 1991; 100: $607-612$.

5. Gravil JH, Al-Rawas OA, Cotton MM, Flanigan U, Irwin A, Stevenson RD. Home treatment of exacerbations of chronic obstructive pulmonary disease by an acute respiratory assessment service. Lancet 1998; 351: $1853-1855$.

6. Skwarska E, Cohen G, Skwarski KM, et al. Randomised controlled trial of supported discharge in patients with exacerbations of chronic obstructive pulmonary disease. Thorax 2000; 55: 907-912.

7. Cotton MM, Bucknall CE, Dagg $\mathrm{K}$, et al. Early discharge for patients with exacerbations of chronic obstructive pulmonary disease: a randomised controlled trial. Thorax 2000; 55: $902-906$.

8. Roca J, Sanchis J, Agustí-Vidal A, et al. Spirometric reference values for a mediterranean population. Bull Eur Physiopathol Respir 1986; 22: 217-224.

9. Killen J, Ellis H. Assisted discharge for patients with exacerbation of chronic obstructive pulmonary disease: save and effective. Thorax 2000; 55: 885.

10. Burge PS, Calverley PM, Jones PW, Spencer SA, Anderson JA, Maslen TK. Randomised, double blind, placebo controlled study of fluticasone propionate in patients with moderate to severe chronic obstructive pulmonary disease: the isolde trial. $B M J$ 2000; 320: $1297-1303$. 